\title{
THE BAIRE CLASS OF APPROXIMATE SYMMETRIC DERIVATES
}

\author{
LEE LARSON
}

\begin{abstract}
It is shown that all approximate symmetric derivatives of measurable functions are in Baire class one. Further, if $f$ is a measurable function which is finite a.e., then its upper and lower approximate symmetric derivates are in Baire class three.
\end{abstract}

An extended real-valued function, $f$, defined on $\mathbf{R}$ is said to be in the first Baire class, abbreviated $\mathscr{B}_{1}$, if there is a sequence of continuous functions, $f_{1}, f_{2}, \ldots$, such that $f_{n}(x) \rightarrow f(x)$ for all $x$. In a similar way, $f$ is in the second Baire class, abbreviated $\mathscr{B}_{2}$, if there is a sequence of functions, $f_{1}, f_{2}, \ldots$, each in $\mathscr{B}_{1}$, such that $f_{n}(x) \rightarrow f(x)$ for all $x$. It is clear that this process can be continued to generate a class of functions, $\mathscr{B}_{\alpha}$, corresponding to each ordinal number $\alpha$ (see Goffman [1]). Our purpose here is to show that the upper and lower approximate symmetric derivates of any measurable function which is finite a.e. are in $\mathscr{B}_{3}$ and if it is symmetrically differentiable, then its approximate symmetric derivative is in $\mathscr{B}_{1}$.

Let $A \subset \mathbf{R}$. The reflection of $A$ through some $x \in \mathbf{R}$ will be denoted $R_{x}(A)$. If $A$ is measurable, then its measure is denoted by $|A|$. The upper density of $A$ at $x \in \mathbf{R}$ is written

$$
\bar{d}(A, x)=\limsup _{h, k \rightarrow 0+} \frac{|A \cap(x-h, x+k)|}{h+k} ;
$$

the lower density of $A$ at $x, \underline{d}(A, x)$, and the density of $A$ at $x, d(A, x)$, are written similarly.

Let $f$ be a measurable function defined on $\mathbf{R}$. If $x, t \in \mathbf{R}$ such that $t \neq 0$ and $f(x+t)-f(x-t)$ is defined, then we write

$$
q(x, t)=\frac{f(x+t)-f(x-t)}{2 t} .
$$

The upper approximate symmetric derivate of $f$ at $x$ is

$$
\bar{f}_{\mathrm{ap}}^{s}(x)=\inf \{a: \bar{d}(\{t: q(x, t)>a\}, 0)=0\} ;
$$

the lower approximate symmetric derivate of $f$ at $x, f_{\mathrm{ap}}^{s}(x)$, is defined analogously. If $\bar{f}_{\text {ap }}^{s}(x)=f_{\text {ap }}^{s}(x)$, whether finite or infinite, then $f$ is approximately symmetrically differentiable at $x$ and the common value of the derivates is written $f_{\text {ap }}^{s}(x)$.

Received by the editors January 1, 1982.

1980 Mathematics Subject Classification. Primary 26A24.

Kev words and phrases. Symmetric derivative, approximate derivative, Baire classes. 
Lemma. Let $f$ be a finite-valued measurable function defined on $\mathbf{R}$. If $\alpha>0, \beta \in \mathbf{R}$ and $\gamma \in(0,1)$, then the sets

$$
A_{f}(\alpha, \beta, \gamma)=\{x:|\{t \in(0, \alpha): q(x, t)>\beta\}|>\alpha \gamma\}
$$

and

$$
B_{f}(\alpha, \beta, \gamma)=\{x:|\{t \in(0, \alpha): q(x, t)<\beta\}|>\alpha \gamma\}
$$

are both open.

Proof. No generality is lost with the assumption that $\beta=0$, for if $\beta \neq 0$ we may consider $g(x)=f(x)-\beta x$ in which case

$$
A_{f}(\alpha, \beta, \gamma)=A_{g}(\alpha, 0, \gamma) \text {. }
$$

Suppose $0 \in A_{f}(\alpha, 0, \gamma)$ and let $C$ be the set of all $t \in(0, \alpha)$ such that:

(i) $f$ is approximately continuous at $t$ and $-t$ and

(ii) $f(t)>f(-t)$.

Then $|C|>\alpha \gamma$ because of the definition of $A_{f}(\alpha, 0, \gamma)$ and because the measurability of $f$ implies that it is approximately continuous a. e. (see Goffman [1, p. 190]). For each positive integer, $n$, define

$$
C_{n}=\left\{t \in C: f(t)-f(-t)>\frac{1}{n}\right\} .
$$

It is clear that $C_{n}$ is measurable for each $n$ and that $C=\cup_{n=1}^{\infty} C_{n}$. Therefore there exists an integer, $m$, large enough so that

$$
\left|C_{m}\right|>\alpha \delta>\alpha \gamma
$$

for some $\delta>\gamma$. Since $f$ is approximately continuous at $t$ and $-t$ whenever $t \in C_{m}$, we may choose $r(t)>0$ such that when $0<h<r(t)$

$$
\left|\left\{x \in[t-h, t+h]:|f(x)-f(t)|<\frac{1}{2 m}\right\}\right|>2 h\left(\frac{3 \gamma+5 \delta}{8 \delta}\right)
$$

and

$$
\left|\left\{x \in[-t-h,-t+h]:|f(x)-f(t)|<\frac{1}{2 m}\right\}\right|>2 h\left(\frac{3 \gamma+5 \delta}{8 \delta}\right) .
$$

Define

$$
\Lambda=\left\{[t-h, t+h]: t \in C_{m}, 0<h<\min \{r(t), t, \alpha-t\}\right\} .
$$

$\Lambda$ is a Vitali cover for $C_{m}$, so there exists a sequence, $\left\{I_{n}\right\} \subset \Lambda$, such that $I_{\mu} \cap I_{\nu}=\varnothing$ whenever $\mu \neq \nu$ and

$$
\left|C_{m}-\bigcup_{n=1}^{\infty} I_{n}\right|=0 .
$$

Thus, we may choose an integer, $N$, large enough so that

$$
\left|\bigcup_{n=1}^{N} I_{n}\right|>\alpha \delta .
$$

Define $t_{n}$ to be the center of $I_{n}, J_{n}=R_{0}\left(I_{n}\right)$,

$$
R_{n}=\left\{t \in I_{n}:\left|f(t)-f\left(t_{n}\right)\right|<\frac{1}{2 m}\right\}
$$


and

$$
S_{n}=\left\{t \in J_{n}:\left|f(t)-f\left(-t_{n}\right)\right|<\frac{1}{2 m}\right\} .
$$

According to (1) and (2),

$$
\left|R_{n}\right|>\left|I_{n}\right| \frac{3 \gamma+5 \delta}{8 \delta} \text { and }\left|S_{n}\right|>\left|J_{n}\right| \frac{3 \gamma+5 \delta}{8 \delta} \text {. }
$$

Note that if $u \in R_{n}$ and $v \in S_{n}$ for some fixed $n$, then

$$
\begin{aligned}
f(u)-f(v) & >\left(f\left(t_{n}\right)-\frac{1}{2 m}\right)-\left(f\left(-t_{n}\right)+\frac{1}{2 m}\right) \\
& =f\left(t_{n}\right)-f\left(-t_{n}\right)-\frac{1}{m}>0
\end{aligned}
$$

because $t_{n} \in C_{m}$.

Choose $\varepsilon$ such that

$$
0<\varepsilon<\frac{\delta-\gamma}{8 \delta} \min _{1 \leqslant n \leqslant N}\left|I_{n}\right|
$$

and let $|x|<\varepsilon$. Then, using (4) and (3) we see that

$$
\begin{aligned}
&|\{t \in(0, \alpha): f(x+t)>f(x-t)\}| \geqslant\left|\bigcup_{n=1}^{N} R_{x}\left(S_{n}\right) \cap R_{n}\right| \\
& \quad=\sum_{n=1}^{N}\left|R_{x}\left(S_{n}\right) \cap R_{n}\right| \\
& \geqslant \sum_{n=1}^{N}\left[\left|R_{x}\left(S_{n}\right)\right|+\left|R_{n}\right|-2|x|-\left|I_{n}\right|\right] \\
&>\sum_{n=1}^{N}\left[\left|J_{n}\right|\left(\frac{3 \gamma+5 \delta}{8 \delta}\right)+\left|I_{n}\right|\left(\frac{3 \gamma+5 \delta}{8 \delta}\right)-\left(\frac{\delta-\gamma}{4 \delta}\right)\left|I_{n}\right|-\left|I_{n}\right|\right] \\
&=\frac{\gamma}{\delta} \sum_{n=1}^{N}\left|I_{n}\right|>\alpha \gamma .
\end{aligned}
$$

Therefore $x \in A_{f}(\alpha, 0, \gamma)$ and it follows that $(-\varepsilon, \varepsilon) \subset A_{f}(\alpha, 0, \gamma)$. If $s \in A_{f}(\alpha, 0, \gamma)$, then by considering $g(x)=f(x+s)$ it can be shown in a manner similar to the above that there is an $\varepsilon>0$ such that $(s-\varepsilon, s+\varepsilon) \subset A_{f}(\alpha, 0, \gamma)$. Therefore $A_{f}(\alpha, 0, \gamma)$ is open.

The proof that $B_{f}(\alpha, \beta, \gamma)$ is open follows from the observation that $B_{f}(\alpha, \beta, \gamma)=$ $A_{-f}(\alpha,-\beta, \gamma)$.

THEOREM 1. If $f$ is a measurable function such that $f_{\mathrm{ap}}^{s}$ exists everywhere, then $f_{\text {ap }}^{s} \in \mathscr{Q B}_{1}$.

Proof. Let $N^{+}=\{x: f(x)=\infty\}$ and $N^{-}=\{x: f(x)=-\infty\}$. If $\left|N^{+}\right|>0$, then there is an $x \in \mathbf{R}$ such that $d\left(N^{+}, x\right)=1$. It follows easily that

$$
d(\{t: q(x, t) \text { is defined }\}, 0)=0
$$


which implies that $f_{\mathrm{ap}}^{s}(x)=-\infty$ and $\bar{f}_{\mathrm{ap}}^{s}(x)=\infty$. But, $f_{\mathrm{ap}}^{s}(x)$ exists so this contradiction leads us to conclude that $\left|N^{+}\right|=0$. It can be shown similarly that $\left|N^{-}\right|=0$. Therefore $f$ is finite a.e. Since the definition of $f_{\mathrm{ap}}^{s}(x)$ does not depend upon $f(x)$, no generality is lost with the assumption that $f$ is real-valued everywhere.

Suppose $\beta \in \mathbf{R}, \gamma \in\left(\frac{1}{2}, 1\right), m$ is a positive integer and

$$
x \in \bigcap_{k=1}^{\infty} \bigcup_{n=k}^{\infty} A_{f}\left(\frac{1}{n}, \beta-\frac{1}{m}, \gamma\right) .
$$

Then there is a sequence of integers, $n_{1}, n_{2}, \ldots$, such that $x \in A_{f}\left(\frac{1}{n_{1}}, \beta-\frac{1}{m_{1}}, \gamma\right)$ for each $i$. Thus,

$$
\left|\left\{t \in\left(0, \frac{1}{n_{i}}\right): q(x, t)>\beta-\frac{1}{m}\right\}\right|>\frac{\gamma}{n_{i}}
$$

and it follows at once that

$$
\bar{d}\left(\left\{t: q(x, t)>\beta-\frac{1}{m}\right\}, 0\right) \geqslant \gamma>\frac{1}{2} .
$$

From the definition of $f_{\mathrm{ap}}^{s}$ we see that $f_{\mathrm{ap}}^{s}(x)>\beta-\frac{1}{m}$. Therefore

$$
\bigcap_{k=1}^{\infty} \bigcup_{n=k}^{\infty} A_{f}\left(\frac{1}{n}, \beta-\frac{1}{m}, \gamma\right) \subset\left\{x: f_{\mathrm{ap}}^{s}(x)>\beta-\frac{1}{m}\right\},
$$

which implies

$$
\bigcap_{m=1}^{\infty} \bigcap_{k=1}^{\infty} \bigcup_{n=k}^{\infty} A_{f}\left(\frac{1}{n}, \beta-\frac{1}{m}, \gamma\right) \subset\left\{x: f_{\mathrm{ap}}^{s}(x) \geqslant \beta\right\} .
$$

Now let $f_{\mathrm{ap}}^{s}(x) \geqslant \beta$. Then for each positive integer, $m$, there is a positive integer, $N$, such that when $n \geqslant N$,

$$
\left|\left\{t \in\left(0, \frac{1}{n}\right): q(x, t)>\beta-\frac{1}{m}\right\}\right|>\frac{\gamma}{n} .
$$

Therefore

$$
x \in \bigcup_{n=k}^{\infty} A_{f}\left(\frac{1}{n}, \beta-\frac{1}{m}, \gamma\right)
$$

for all $k$. This implies that

$$
x \in \bigcap_{k=1}^{\infty} \bigcup_{n=k}^{\infty} A_{f}\left(\frac{1}{n}, \beta-\frac{1}{m}, \gamma\right) .
$$

Since $f_{\mathrm{ap}}^{s}(x) \geqslant \beta$, this same reasoning holds for any positive integer, $m$, and so

$$
x \in \bigcap_{m=1}^{\infty} \bigcap_{k=1}^{\infty} \bigcup_{n=k}^{\infty} A_{f}\left(\frac{1}{n}, \beta-\frac{1}{m}, \gamma\right) .
$$

In light of (5) and (6) we conclude that

$$
\left\{x: f_{\mathrm{ap}}^{s}(x) \geqslant \beta\right\}=\bigcap_{m=1}^{\infty} \bigcap_{k=1}^{\infty} \bigcup_{n=k}^{\infty} A_{f}\left(\frac{1}{n}, \beta-\frac{1}{m}, \gamma\right) .
$$


It follows similarly that

$$
\left\{x: f_{\mathrm{ap}}^{s}(x) \leqslant \beta\right\}=\bigcap_{m=1}^{\infty} \bigcap_{k=1}^{\infty} \bigcup_{n=k}^{\infty} B_{f}\left(\frac{1}{n}, \beta+\frac{1}{m}, \gamma\right) .
$$

Now we apply the lemma to see that both

$$
\left\{x: f_{\text {ap }}^{s}(x) \geqslant \beta\right\} \text { and }\left\{x: f_{\text {ap }}^{s}(x) \leqslant \beta\right\}
$$

are $G_{\delta}$ sets. Using the standard theorem in Goffman [1, p. 141], it follows that $f_{\text {ap }}^{s} \in \mathscr{B}_{1}$.

THEOREM 2. Let $f$ be a measurable function which is real-valued $a$. $e$. and let $\beta \in[-\infty, \infty]$. Then the sets

$$
\left\{x: \bar{f}_{\text {ap }}^{s}(x)>\beta\right\} \text { and }\left\{x: \underline{f a p}_{\text {ap }}^{s}(x)<\beta\right\}
$$

are both $G_{\delta \sigma}$ sets and the sets

$$
\left\{x: \bar{f}_{\mathrm{ap}}^{s}(x)<\beta\right\} \text { and }\left\{x: f_{\mathrm{ap}}^{s}(x)>\beta\right\}
$$

are both $F_{\sigma \delta \sigma}$ sets.

PROOF. As before we may assume that $f$ is real-valued everywhere. First, assume $\beta \in \mathbf{R}$. Let $\gamma \in(0,1)$ and

$$
x \in \bigcap_{k=1}^{\infty} \bigcup_{0<\alpha<1 / k} A_{f}(\alpha, \beta, \gamma) .
$$

Then for each positive integer, $k$, there is an $\alpha_{k} \in\left(0, \frac{1}{k}\right)$ such that

$$
\left|\left\{t \in\left(0, \alpha_{k}\right): q(x, t)>\beta\right\}\right|>\alpha_{k} \gamma .
$$

Therefore

$$
\bar{d}(\{t: q(x, t)>\beta\}, 0) \geqslant y
$$

and we conclude that $\bar{f}_{\mathrm{ap}}^{s}(x)>\beta$. From this it follows that if

$$
x \in \bigcup_{n=2}^{\infty} \bigcap_{k=1}^{\infty} \bigcup_{0<\alpha<1 / k} A_{f}\left(\alpha, \beta, \frac{1}{n}\right),
$$

then $\bar{f}_{\mathrm{ap}}^{s}(x)>\beta$. Thus

$$
\bigcup_{n=2}^{\infty} \bigcap_{k=1}^{\infty} \bigcup_{0<\alpha<1 / k} A_{f}\left(\alpha, \beta, \frac{1}{n}\right) \subset\left\{x: \bar{f}_{\mathrm{ap}}^{s}(x)>\beta\right\} .
$$

Now, suppose $\bar{f}_{\text {ap }}^{s}(x)>\beta$. Then there is a positive integer, $n_{0}$, such that

$$
\bar{d}(\{t: q(x, t)>\beta\}, 0)>\frac{1}{n_{0}} .
$$

This implies that for each positive integer, $k$, there is an $\alpha_{k} \in\left(0, \frac{1}{k}\right)$ such that $x \in A_{f}\left(\alpha_{k}, \beta, \frac{1}{n_{0}}\right)$. Therefore

$$
x \in \bigcap_{k=1}^{\infty} \bigcup_{0<\alpha<1 / k} A_{f}\left(\alpha, \beta, \frac{1}{n_{0}}\right) \subset \bigcup_{n=2}^{\infty} \bigcap_{k=1}^{\infty} \bigcup_{0<\alpha<1 / k} A_{f}\left(\alpha, \beta, \frac{1}{n}\right)
$$


and so

$$
\left\{x: \bar{f}_{\mathrm{ap}}^{s}(x)>\beta\right\} \subset \bigcup_{n=2}^{\infty} \bigcap_{k=1}^{\infty} \bigcup_{0<\alpha<1 / k} A_{f}\left(\alpha, \beta, \frac{1}{n}\right) .
$$

From (7) and (8) it follows that

$$
\left\{x: \bar{f}_{\mathrm{ap}}^{s}(x)>\beta\right\}=\bigcup_{n=2}^{\infty} \bigcap_{k=1}^{\infty} \bigcup_{0<\alpha<1 / k} A_{f}\left(\alpha, \beta, \frac{1}{n}\right) .
$$

It follows from the lemma that $\left\{x: \bar{f}_{\text {ap }}^{s}(x)>\beta\right\}$ is a $G_{\delta \sigma}$ set.

If $|\beta|=\infty$, then

$$
\left\{x: \bar{f}_{\mathrm{ap}}^{s}(x)>\infty\right\}=\varnothing \text { and }\left\{x: \bar{f}_{\mathrm{ap}}^{s}(x)>-\infty\right\}=\bigcup_{n=1}^{\infty}\left\{x: \bar{f}_{\mathrm{ap}}^{s}(x)>-n\right\}
$$

are both $G_{\delta \sigma}$ sets. Note that if $\beta \in \mathbf{R}$, then

$$
\begin{aligned}
& \left\{x: \bar{f}_{\text {ap }}^{s}(x) \geqslant \beta\right\}=\bigcap_{n=1}^{\infty}\left\{x: \bar{f}_{\text {ap }}^{s}(x)>\beta-\frac{1}{n}\right\}, \\
& \left\{x: \bar{f}_{\text {ap }}^{s}(x) \geqslant \infty\right\}=\bigcap_{n=1}^{\infty}\left\{x: \bar{f}_{\text {ap }}^{s}(x)>n\right\}
\end{aligned}
$$

and

$$
\left\{x: \bar{f}_{\text {ap }}^{s}(x) \geqslant-\infty\right\}=\mathbf{R},
$$

where all three sets are $G_{\delta \sigma \delta}$ sets. Therefore, for any $\beta \in[-\infty, \infty]$,

$$
\left\{x: \bar{f}_{\text {ap }}^{s}(x)<\beta\right\}=\mathbf{R}-\left\{x: \bar{f}_{\text {ap }}^{s}(x) \geqslant \beta\right\}
$$

is an $F_{\sigma \delta \sigma}$ set.

To prove the theorem for the lower derivate, it suffices to note that if $g=-f$, then $f_{\text {ap }}^{s}=-\left(\bar{g}_{\text {ap }}^{s}\right)$.

According to Goffman [1, p. 141], Theorem 2 immediately implies the following corollary.

COROLlaRY 3. Let $f$ be a measurable function which is finite a.e. Then $\bar{f}_{\mathrm{ap}}^{s}$ and $f_{\mathrm{ap}}^{s}$ are both in $\mathbb{B}_{3}$.

Corollary 3 is an improvement on a theorem of Kundu [2], who showed that if $f$ is continuous, then $\bar{f}_{\text {ap }}^{s}$ is measurable. It was shown by Preiss [4] that all ordinary approximate derivatives are in $9_{1}$ and Larson [3] showed that all ordinary symmetric derivatives are in ${ }_{9} B_{1}$; Theorem 1 is a partial extension of both of these results.

\section{REFERENCES}

1. C. Goffman, Real functions, Holt, New York, 1964

2. N. K. Kundu, Symmetric derivatives and approximate symmetric derivatives of continuous functions, Rev. Roumaine Math. Pures Appl. 18 (1973). 689-697.

3. L. Larson. The symmetric derivatives. Trans. Amer. Math Soc. (to appear).

4. D. Preiss, Approximate derivatives and Baire classes, Czechoslovak Math. J. 21 (96) (1971), 373-382. 